\title{
CONSTRUCTAL TREE-SHAPED WATER DISTRIBUTION NETWORKS BY AN ENVIRONMENTAL APPROACH
}

\author{
P. BIEUPOUDE ${ }^{1}$, Y. AZOUMAH ${ }^{1} \&$ P. NEVEU ${ }^{2}$ \\ ${ }^{1}$ Laboratoire Energie Solaire et Economie d'Energie (LESEE), Fondation 2iE 01 BP 594, Ouagadougou 01, Burkina Faso. \\ ${ }^{2}$ Laboratoire PROcédés, Matériaux et Energie Solaire (PROMES-CNRS, UPR 8521), Rambla de la thermodynamique, \\ Tecnosud, 66100 Perpignan, France.
}

\begin{abstract}
Flow systems most often present thermal, mechanical and chemical losses due to irreversibility during the flowing fluid transport. These losses strongly impact both their energy performances and the flowing fluid quality. In this paper, two effective, drinking water and irrigation, tree-shaped networks (from the fluid quality and energy performance points of view) are constructed by using the constructal approach coupled with the exergy destruction minimization method. It is shown that in the construction of tree-shaped network for water distribution, the method of exergy destruction minimization is equivalent to minimizing mechanical irreversibility (this is equivalent to pumping power) under a water quality constraint. For both phenomena occurring in the network (energy consumption and the fluid quality degradation), this study offers new interesting routes for optimizing the system either by the exergy destruction minimization (in that case, both irreversible processes are taken into account in the design procedure) or by minimizing one of the two irreversible processes, the other being taken into account as the design constraint. The originality of the method relies on the introduction of the environmental protection through the control of the flowing fluid quality. This paper shows that, for the performance improvement of a water distribution network, it is important to focus on the design of the network rather than enhancing only the transport properties. Note finally that the focus on the quality in flow systems is a crucial approach in environmental engineering such as drinking water distribution systems or chemical fluids transfer systems. The approach presented in this paper should be seen as an introduction to reactive flow systems designing by constructal approach.
\end{abstract}

Keywords: constructal theory, design, drinking water, exergy, irrigation, water distribution network.

\section{INTRODUCTION}

The need of new scientifically based approaches with a special care of the environment by quantitative or qualitative optimization is very critical today in different fields of engineering. In hydraulics, water distribution systems involve tree-shaped networks as well as several other areas of engineering such as electronic components cooling [1], heat and mass transfer in a solid-gas reactive porous media [2] or most generally flow structures [3, 4]. The effective implementation of these flow architectures relies on their irreversibility minimization. For the specific case of pressure water distribution systems, two important phenomena are in competition: head losses leading to energy consumption and chemical losses causing water quality degradation, according to Kerneïs et al. [5], showing that there is a trade-off to be found by design procedures. This is the concern of this paper which is an extended version of our recent works [6] in which thermodynamics of irreversible process is used [7] to describe the flow. This enabled to quantify the total exergy destruction of the system. Furthermore, a readjustment of this thermodynamical approach is made to account very well that the impacts of water distribution network design on water quality degradation (which is directly linked to human health). Constructal design is then performed, on one hand, by optimizing the total exergy destruction, and on the other, by minimizing one of the two irreversible processes while the second is considered as the design constraint. Results are later analyzed from irrigation and drinking water constraints points of view. 


\section{THERMODYNAMIC ANALYSIS OF A PRESSURIZED FLOW}

This section aims to describe and set thermodynamics balances of the flow under pressure in a pipe.

\subsection{Description of the system}

From many applied hydraulics literature [8-10], it is known that head losses in a pipe (Fig. 1) (caused by mechanical resistances) can be expressed by:

$$
\Delta H=a \frac{Q^{n}}{D^{m}} L
$$

where $a$ is a dimensionless constant depending on the pipe material, $Q$ is the volumetric flow rate $\left(\mathrm{m}^{3} / \mathrm{s}\right), L$ is the pipe length $(\mathrm{m})$ and $D$ its diameter $(\mathrm{m})$.

It is also known that the kinetic of chemical reaction in drinking water pipe can be considered as a first order kinetic close to the ones met in the cases of water treatments reagents like chlorine residual which is generally modeled by a first order kinetic [11].

More precisely, the variation of the concentration of the main reagent obeys to the following law:

$$
C(t)=C_{0} e^{-k t}
$$

where $C_{0}$ is the initial concentration at the input point of the pipe; $k$ is the reagent decay (/s). Thus, the variation of the concentration between the input and output points can be defined by:

$$
\Delta C=C_{0}-C(t)=C_{0}\left(1-e^{-k t}\right)
$$

where $t$ is the residence time that can be linked to the volumetric flow rate and the diameter by:

$$
t=\frac{\pi D^{2} L}{4 Q}
$$

If we substitute $t$ by its expression eqn (3), it results in the equation of the variation of the reagent concentration:

$$
\Delta C=C_{0}\left(1-e^{-k \frac{\pi D^{2} L}{4 Q}}\right)
$$

Figures 2-4 show the evolution of mechanical and chemical phenomena in the pipe for the following parameters: PVC pipe; Hazen William head losses formula parameters for PVC pipe given by Carlier [8] and Lahiouel et al. [9]: $a=0.00099597, n=1.852, m=4.87$. In Fig. 2, it is shown that head losses $(\Delta H)$ and the concentration decay $(\Delta C)$ have antagonist evolution over the volumetric flow rate. In fact, head losses increase with the flow rate while the concentration decay decreases with it. For $Q=0.05 \mathrm{~m}^{3} / \mathrm{s}$ and $L=300 \mathrm{~m}$, the both curves are also antagonist as shown in Fig. 3 with a diminution of $\Delta H$ while the concentration decay is increasing. But keeping $D$ and $Q$ as constants and the length $L$ variable, we notice as shown in Fig. 4, that $\Delta H$ and the concentration decay evolve quasi-linearly with the pipe length.

In sum, for a given diameter and flow rate, $\Delta H$ and $\Delta C$ increase with the pipe length (Fig. 4). Yet Figs 2 and 3 reveal an antagonist evolution of $\Delta H$ and $\Delta C$ with $D$ or $Q$. Therefore, one can conclude 


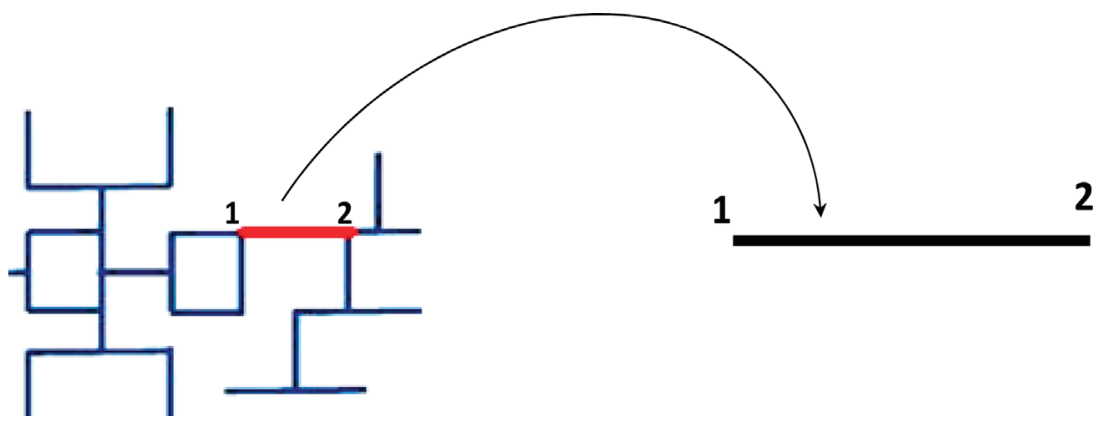

Figure 1: A given pipe of a water distribution network.

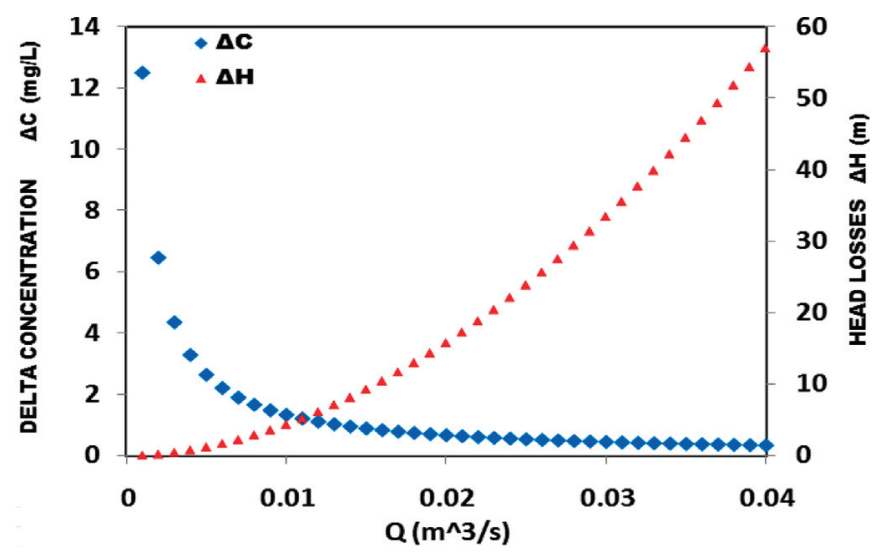

Figure 2: Evolution of head losses and the concentration decay over the flow rate.

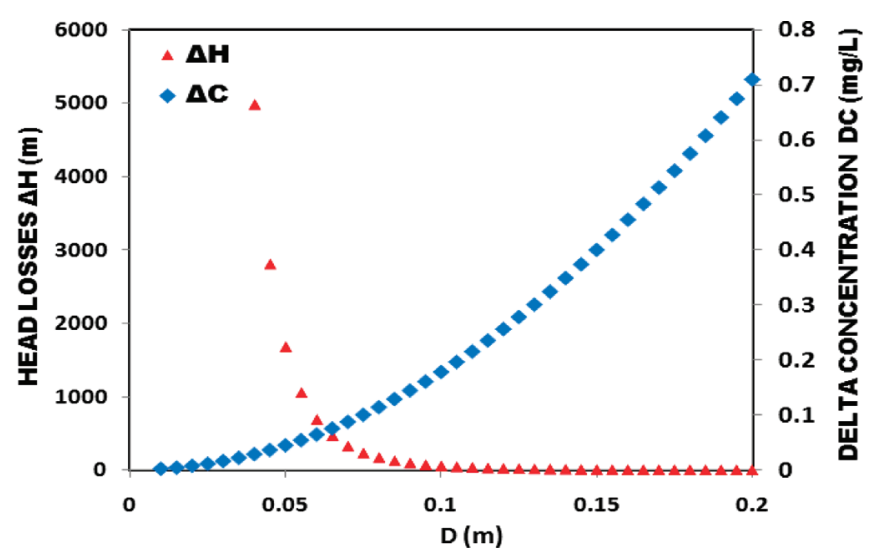

Figure 3: Evolution of head losses and the concentration decay over the diameter. 


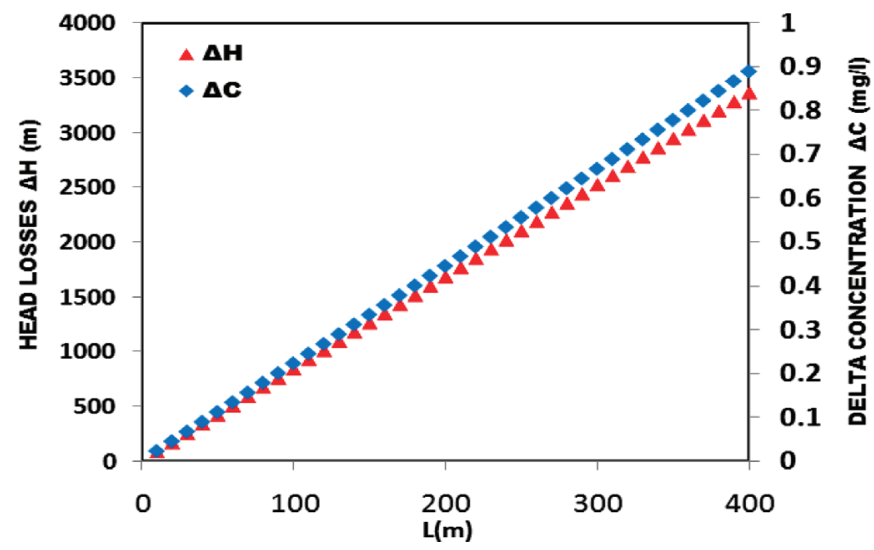

Figure 4: Evolution of head losses and the concentration decay over the pipe length.

that the pipe length does not affect in different way irreversibilities in the pipe while the diameter and the flow rate are the most sensible parameters.

The antagonist curves in Figs 2 and 3 show obviously that there is a trade-off to be determined between the chemical and the mechanical phenomena. But these two phenomena are not comparable in sense that mechanical irreversibilities describe quantity and chemical ones deals with quality. Setting thermodynamic balances, as exposed in exergy destruction or entropy generation papers of Prigogine [7] and Azoumah et al. [2], can enable to take into account the contribution of all the phenomena occurring in the system.

\subsection{Thermodynamic balances of the flow}

2.2.1 Assumptions and transient balances: mass, enthalpy and exergy Consider at a very simple scale the model of reaction occurring in the flow to be described as follow:

$$
A+B \rightarrow C+D
$$

$C$ and $A$ are reagents while $B$ and $D$ are the products of the reaction.

We have:

$$
\begin{aligned}
& \frac{d N_{H_{2} O}}{d t}=\dot{n}_{1} \cdot\left(1-y_{C_{1}}-y_{A 1}-y_{B 1}-y_{D 1}\right)-\dot{n}_{2} \cdot\left(1-y_{C_{2}}-y_{A 2}-y_{B 2}-y_{D 2}\right) \\
& \frac{d E}{d t}=\dot{n}_{1} \cdot h_{1}-\dot{n}_{2} \cdot h_{2}-\dot{q}_{0} \\
& \frac{d\left(E-T_{0} S\right)}{d t}=\dot{n}_{1} \cdot e x_{1}-\dot{n}_{2} \cdot e x_{2}-\dot{q}_{0}(0)-e \dot{x}_{d}
\end{aligned}
$$

where $\dot{n}_{i}$ : molar flow rate (mol/s), $y_{i}$ : molar fraction of the $i$ constituent $(-), h_{i}$ : total molar enthalpy of the solution $(\mathrm{J} / \mathrm{mole})$, ex $x_{i}$ : molar exergy of the solution $(\mathrm{J} / \mathrm{mole}), e x_{d}$ : exergy destruction in the system $(\mathrm{J} / \mathrm{mole}), \dot{q}_{0}$ : heat losses with surrounding at $T_{0}$ temperature $(\mathrm{W}), k$ : constituent $c_{1}$ decay constant (/s), 1: input and 2: output. 
By assuming a steady state and very weak concentrations of reactants and products $\left(y_{i}<<1\right)$, eqn (7) we have:

$$
\begin{aligned}
& \dot{n}_{1} \approx \dot{n}_{2} \\
& \dot{q}_{0}=\dot{n}_{1} \cdot\left(h_{1}-h_{2}\right) \\
& e \dot{x}_{d}=\dot{n}_{1} \cdot\left(e x_{1}-e x_{2}\right)
\end{aligned}
$$

2.2.2 Thermodynamic analysis of the flowing fluid

Molar enthalpy of the solution: The total molar enthalpy of the fluid is expressed by eqn (9):

$$
h=h(T, p, y)+M \frac{V^{2}}{2}+g z
$$

where $h(T, p, y)$ is the molar enthalpy of the solution ( $\mathrm{J} / \mathrm{mole}), M$ is the molar mass of the solution defined by:

$$
\begin{aligned}
M= & y_{C} M_{C}+y_{A} M_{A}+y_{B} M_{B}+y_{D} M_{D} \\
& +\left(1-y_{A}-y_{B}-y_{C}-y_{D}\right) M_{H_{2} O} \\
\cong & M_{H_{2} O}
\end{aligned}
$$

$V$ is the flow velocity in the pipe $(\mathrm{m} / \mathrm{s}), g$ is the gravitational acceleration $\left(\mathrm{m} / \mathrm{s}^{2}\right)$ and $z$ is the altitude (m). As concentrations of reactants and products are supposed to be small (diluted solution), the molar enthalpy is then:

$$
\begin{aligned}
h(T, p, y)= & \left(1-y_{C}-y_{A}-y_{B}-y_{D}\right) \cdot h_{H_{2} O}(T, p)+y_{C} \cdot h_{C}(T, p) \\
& +y_{A} \cdot h_{A}(T, p)+y_{B} \cdot h_{B}(T, p)+y_{D} \cdot h_{D}(T, p)
\end{aligned}
$$

where $h_{\mathrm{H}_{2} \mathrm{O}}(T, p), h_{C}(T, p), h_{A}(T, p), h_{B}(T, p)$ and $h_{D}(T, p)$ are molar partial enthalpies of constituents. With the consideration that $y_{\mathrm{i}}<<1$, the eqn (11) can be approximated by:

$$
h(T, p, y) \approx h_{H_{2} O}(T, p)
$$

Besides, considering that it is an ideal and incompressible solution $\left(v_{\mathrm{H}_{2} \mathrm{O}}=\right.$ cste), we finally have:

$$
h(T, p, y) \approx h_{\mathrm{H}_{2} \mathrm{O}}^{0}(T, p)+c_{\mathrm{H}_{2} \mathrm{O}} \cdot\left(T-T^{0}\right)+v_{\mathrm{H}_{2} \mathrm{O}} \cdot\left(p-p^{0}\right)
$$

where $C_{\mathrm{H}_{2} \mathrm{O}}$ and $v_{\mathrm{H}_{2} \mathrm{O}}$ are respectively the molar thermal capacity and the molar volume of the water. 0 represents the reference state.

Molar entropy: Molar entropy of an ideal solution can be expressed by:

$$
\begin{aligned}
s(T, p, y) & =\left(1-y_{A}-y_{B}-y_{C}-y_{D}\right) \cdot\left[s_{H_{2} O}(T, p)-R \cdot \operatorname{Ln}\left(1-y_{A}-y_{B}-y_{C}-y_{D}\right)\right] \\
& +y_{A} \cdot\left[s_{A}(T, p)-R \cdot \operatorname{Ln}\left(y_{A}\right)\right]+y_{B} \cdot\left[s_{B}(T, p)-R \cdot \operatorname{Ln}\left(y_{B}\right)\right] \\
& +y_{D} \cdot\left[s_{D}(T, p)-R \cdot \operatorname{Ln}\left(y_{D}\right)+y_{C} \cdot\left[s_{C}(T, p)-R \cdot \operatorname{Ln}\left(y_{C}\right)\right]\right]
\end{aligned}
$$

where $s_{\mathrm{H}_{2} \mathrm{O}}(T, p), s_{A}(T, p), s_{B}(T, p)$ and $s_{C}(T, p)$ are specific molar enthalpies. 
By considering that $y_{i}<<1$, the eqn (14) becomes:

$$
s(T, p, y)=s_{H_{2} O}(T, p)-R \cdot\left[\begin{array}{l}
\left(1-y_{A}-y_{B}-y_{C}-y_{D}\right) \operatorname{Ln}\left(1-y_{A}-y_{B}-y_{C}-y_{D}\right) \\
+y_{A} \operatorname{Ln}\left(y_{A}\right)+y_{B} \operatorname{Ln}\left(y_{B}\right)+y_{C} \operatorname{Ln}\left(y_{C}\right)+y_{D} \operatorname{Ln}\left(y_{D}\right)
\end{array}\right]
$$

And finally, as the solution is supposed to be incompressible, the relation (15) can be replaced by:

$$
\begin{aligned}
s(T, p, y) \approx & \cdot S_{H_{2} O}^{0}(T, p)+c_{H_{2} O} \cdot \operatorname{Ln}\left(\frac{T}{T^{0}}\right) \\
& -R \cdot\left[\begin{array}{l}
\left(1-y_{A}-y_{B}-y_{C}-y_{D}\right) \operatorname{Ln}\left(1-y_{A}-y_{B}-y_{C}-y_{D}\right) \\
+y_{A} \operatorname{Ln}\left(y_{A}\right)+y_{B} \operatorname{Ln}\left(y_{B}\right)+y_{C} \operatorname{Ln}\left(y_{C}\right)+y_{D} \operatorname{Ln}\left(y_{D}\right)
\end{array}\right]
\end{aligned}
$$

Molar exergy: The molar exergy of the solution can be deduced directly from eqns (9), (13) and (16) as following:

$$
\begin{aligned}
\operatorname{ex}(T, p, y, V, z) \approx & h_{\mathrm{H}_{2} \mathrm{O}}^{0}(T, p)-T^{0} \cdot s_{\mathrm{H}_{2} \mathrm{O}}^{0}(T, p) \\
& +c_{\mathrm{H}_{2} \mathrm{O}} \cdot\left(\left(T-T^{0}\right)-T^{0} \operatorname{Ln} \frac{T}{T^{0}}\right) \\
& +v_{\mathrm{H}_{2} \mathrm{O}} \cdot\left(p-p^{0}\right) \\
& +M \cdot V^{2} / 2 \\
& +g \cdot z \\
& +R \cdot T^{0} \cdot\left[\begin{array}{l}
\left(1-y_{A}-y_{B}-y_{C}-y_{D}\right) \operatorname{Ln}\left(1-y_{A}-y_{B}-y_{C}-y_{D}\right) \\
+y_{A} \operatorname{Ln}\left(y_{A}\right)+y_{B} \operatorname{Ln}\left(y_{B}\right)+y_{C} \operatorname{Ln}\left(y_{C}\right)+y_{D} \operatorname{Ln}\left(y_{D}\right)
\end{array}\right]
\end{aligned}
$$

Formula of the total destruction of the exergy: Finally, the exergy destruction is obtained from previous equations as follows:

$$
e \dot{x}_{d}=\dot{n}_{1} \cdot\left[\begin{array}{l}
c_{H_{2} O} \cdot\left(T_{1}-T_{2}-T^{0} \operatorname{Ln} \frac{T_{1}}{T_{2}}\right) \\
+v_{H_{2} O} \cdot\left(p_{1}-p_{2}\right)+M \cdot\left(V_{1}^{2}-V_{2}^{2}\right) / 2+g \cdot\left(z_{1}-z_{2}\right) \\
-R \cdot T^{0} \cdot \operatorname{Ln}\left[\begin{array}{l}
\left(1-y_{A}-y_{B}-y_{C}-y_{D}\right) \operatorname{Ln}\left(1-y_{A}-y_{B}-y_{C}-y_{D}\right) \\
+y_{A} \operatorname{Ln}\left(y_{A}\right)+y_{B} \operatorname{Ln}\left(y_{B}\right)+y_{C} \operatorname{Ln}\left(y_{C}\right)+y_{D} \operatorname{Ln}\left(y_{D}\right)
\end{array}\right]
\end{array}\right]
$$

The variation of the water temperature is negligible from the input point to the output point $\left(T_{1}=T_{2}\right)$. The velocity is also supposed to be constant, $V_{1}=V_{2}$, therefore the total exergy destruction is:

$$
e \dot{x}_{d}=\dot{n}_{1} \cdot\left[\begin{array}{l}
v_{H_{2} O} \cdot\left(p_{1}-p_{2}\right)+g \cdot\left(z_{1}-z_{2}\right) \\
-R \cdot T^{0} \cdot \operatorname{Ln}\left[\begin{array}{l}
\left(1-y_{A}-y_{B}-y_{C}-y_{D}\right) \operatorname{Ln}\left(1-y_{A}-y_{B}-y_{C}-y_{D}\right) \\
+y_{A} \operatorname{Ln}\left(y_{A}\right)+y_{B} \operatorname{Ln}\left(y_{B}\right)+y_{C} \operatorname{Ln}\left(y_{C}\right)+y_{D} \operatorname{Ln}\left(y_{D}\right)
\end{array}\right]
\end{array}\right]
$$


The first term of this formula is equal to head losses which are evaluated by eqn (1) according to Carlier [8].

Total exergy destruction finally writes:

$$
e \dot{x}_{d}=\dot{n}_{1} \cdot\left[\begin{array}{l}
{\left[\begin{array}{l}
a g \cdot M_{e} \cdot \frac{Q^{n}}{D^{m}} \cdot L
\end{array}\right]} \\
-R \cdot T^{0} \cdot \operatorname{Ln}\left[\begin{array}{l}
\left(1-y_{A}-y_{B}-y_{C}-y_{D}\right) \operatorname{Ln}\left(1-y_{A}-y_{B}-y_{C}-y_{D}\right) \\
+y_{A} \operatorname{Ln}\left(y_{A}\right)+y_{B} \operatorname{Ln}\left(y_{B}\right)+y_{C} \operatorname{Ln}\left(y_{C}\right)+y_{D} \operatorname{Ln}\left(y_{D}\right)
\end{array}\right]
\end{array}\right]
$$

That can be written in contracted form by:

$$
e \dot{x}_{d}=\dot{n}_{1} \cdot\left(\operatorname{ag} \cdot M_{e} \cdot \frac{Q^{n}}{D^{m}} \cdot L-R \cdot T^{0} \cdot \operatorname{Ln}\left[\frac{Y_{2}}{Y_{1}}\right]\right)
$$

where $\left(Y_{2} / Y_{1}\right)$ is the variation of terms with log, eqn (20), from input 1 to output 2.

Equation (21) quantifies, on a same thermodynamic basis, how chemical and mechanical irreversibilities impact the total exergy destruction. The two phenomena can be then assessed, and analyzed.

\subsubsection{Total exergy destruction analysis}

In this section, a large interval of molar fraction is considered to analyze the evolution of the exergy destruction. Consider a pipe of a known length $L(L=200 \mathrm{~m})$ and a known volumetric flow rate $Q$ $\left(Q=0.01 \mathrm{~m}^{3} / \mathrm{s}\right)$ and assuming its diameter as the unknown variable. The WDN operational constraints on velocities (Section 3.2) explained by Carlier [8], to avoid unacceptable deposits in pipes (due to low velocities) or pipes breaks/damages (due to higher velocities) applies for a given pipe (of flow velocity $V$ ) is : $V_{\min }<\mathrm{V}<V_{\max }$. With respect to this, taking $Q=0.01 \mathrm{~m}^{3} / \mathrm{s}$ (for this illustration) is equivalent to selecting maximal and minimal diameter of the pipe. In Fig. 5, the total exergy destruction is calculated for different values of the selected diameter interval.

Two different situations are presented. On one hand, when the molar fraction is high $\left(Y_{1}=6.5 \times\right.$ $10^{-4}, Y_{1}=1.5 \times 10^{-3}$, and $Y_{1}=3.0 \times 10^{-3}$ ), the diameter that leads to the total exergy destruction minimization exists (in this selected diameter interval). On the other hand, for low values of molar fraction under $10^{-4}$ (e.g. $Y_{1}=2.5 \times 10^{-5}$ ), the diameter that minimizes the exergy destruction does not exist in the selected diameter interval (Fig. 5a), but exists for higher diameters (without respect to velocities constraints; Fig. 5b).

This second situation is generally met in drinking water distribution area because of the WHO (World Health Organization) regulation specifications [12] that are applied to ensure water quality.

Indeed, for drinking water, initially treated and put in distribution network, a good concentration of chemicals like chlorine residuals means a good quality of water. It is important to know that the quality agents like chlorine residuals, destroy pathogenic microorganisms which cause typhoid, hepatitis, cholera or bacillary dysentery, according to Crittenden [13], but their concentrations decrease with the residence time until they become completely imperceptible at the end of the pipe according to Powell et al. [14] and Rodriguez et al. [15]. For instance, in USA, the Environmental Protection Agency (USEPA) imposes chlorine residuals concentration closed to $0.2 \mathrm{mg} / \mathrm{l}$ at least in water distribution pipes after water treatment points, as shown by EPA [16]. 

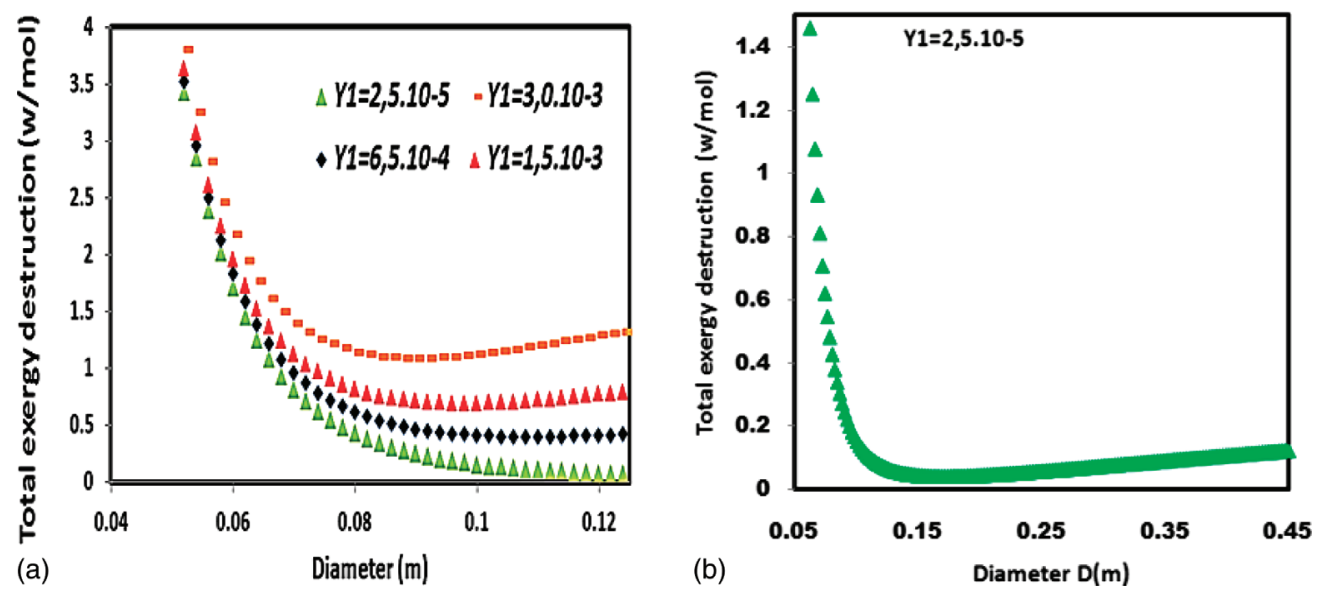

Figure 5: Total exergy destruction for different molar fraction over the pipe diameters selected in the domain of normal velocities (a), exergy destruction admitting minimal point for higher diameters (b).

Though the above result seems to be original, interesting and more adapted for some chemical engineering fields (sanitation, depollution), presented by Torrijos et al. [17] and Montangero et al. [18], it cannot be applied to the field of drinking water networks optimization since molar fractions of constituents in this field, are very low ranging around $10^{-6}$ due to the WHO regulation on chlorine residuals [12]. Therefore, it seems more relevant to readjust and reformulate this above approach to incorporate the criterion of water quality protection, so health care. A new design method based on weighting the different identified irreversibilities has been developed. This new approach has been used to find the permitted velocities interval where the pipe diameter can minimize simultaneously the total amount of irreversibilities of the system.

\section{REFORMULATION OF THE OBJECTIVE FUNCTION BY WEIGHTING IRREVERSIBILITIES}

In order to help in economy saving in infrastructure projects with a special care to the environment, in this section, an improvement of the optimizing approach developed in the previous sections have been performed. The new function built is directly linked to the system dimensions in order to measure effects of the network design on its effectiveness. This new function has been called 'reduced exergy destruction of the system'.

\subsection{Formulation of the objective function}

As shown in eqn (1), for a unit length, mechanical irreversibilities are proportional to $Q^{n} / D^{m}$ according to Carlier [8] and Lehiouel et al. [9] where $n$ and $m$ are friction losses parameters while the water residence time in the pipe, on which depend chemical irreversibilities, is proportional to $D^{2} / Q$.

In this way, the overall irreversibilities created during the flow in the pipe can be assessed by a combination of these two terms as following:

$$
\dot{e x}_{d} \approx \alpha \frac{Q^{n}}{D^{m}}+\beta \frac{D^{2}}{Q}
$$


where $\alpha$ and $\beta$ are constant weights. Now, a new flexible function free from the duct material, describing simultaneously mechanical and chemical phenomena in the pipe is built. By dividing the relation (22) by $a$ (in order to have only one coefficient) we have:

$$
e \dot{x}_{d} \approx \frac{Q^{n}}{D^{m}}+\gamma_{p} \frac{D^{2}}{Q}
$$

where $\gamma_{p}$ is $\beta / \alpha$.

3.2 Limits of $\gamma_{p}$ and study of the objective function

Making $\partial\left(e x_{d}\right) / \partial D=0$ permits to determine the optimal diameter $D_{o p t}$ for a unique pipe flow.

$$
D_{o p t}=\left(\frac{m}{2 \gamma_{p}}\right)^{1 /(m+2)} Q^{\left(\frac{n+1}{m+2}\right)}
$$

This diameter performs the simultaneous minimization of both qualitative and mechanical irreversibilities. Taking into account practice constraints during the implementation, namely maximal and minimal velocities allowed in water distribution [8], a confined interval of the weight $\gamma_{p}$ can be provided. Indeed for a uniform flow, it is known that:

$$
D=\frac{4 Q}{\pi V}
$$

Meaning that, taking into account limit velocities $\left(V_{\min }, V_{\max }\right)$ we have:

$$
\frac{4 Q}{\pi V_{\max }} \leq D_{\text {opt }} \leq \frac{4 Q}{\pi V_{\min }}
$$

And by replacing $D_{o p t}$ by its expression eqn (25), limits of the weight $\gamma_{p}$ can be fixed as:

$$
\left(\frac{\pi V_{\min }}{4}\right)^{(m+2)} \times Q_{o}{ }^{(n-m-1)} \leq \frac{2 \gamma_{p}}{m} \leq\left(\frac{\pi V_{\max }}{4}\right)^{(m+2)} \times Q_{o}{ }^{(n-m-1)}
$$

In Fig. 6, plots of the exergy destruction for various values of $\gamma_{p}$ are shown.

It is revealed for higher values of $\gamma_{p}$ that an optimal diameter exists for minimization of the overall irreversibilities created in the pipe and the corresponding velocities are in the confined interval previously defined in eqns (26) and (27). But for lower values of $\gamma_{p}$, there is no diameter that minimizes simultaneously the amount of irreversibilities created in the pipe for effective values of the velocities. It is then shown that the priority given to energy saving or water quality protection cannot be indefinitely increased, otherwise velocities limits are overtaken.

A sensitiveness study made on the weight $\gamma_{p}$ for different flow rates is illustrated in Fig. 7 showing that, for any flow rate, an optimal diameter performing simultaneously the minimization of the overall irreversibilities in the pipe exists. It means that it is possible to use this approach for any quantity (by the same way) for any size of network.

In this section, associating environmental questions with thermodynamics, we have performed a qualitative and quantitative design tool for drinking water distribution network. Mechanical and 

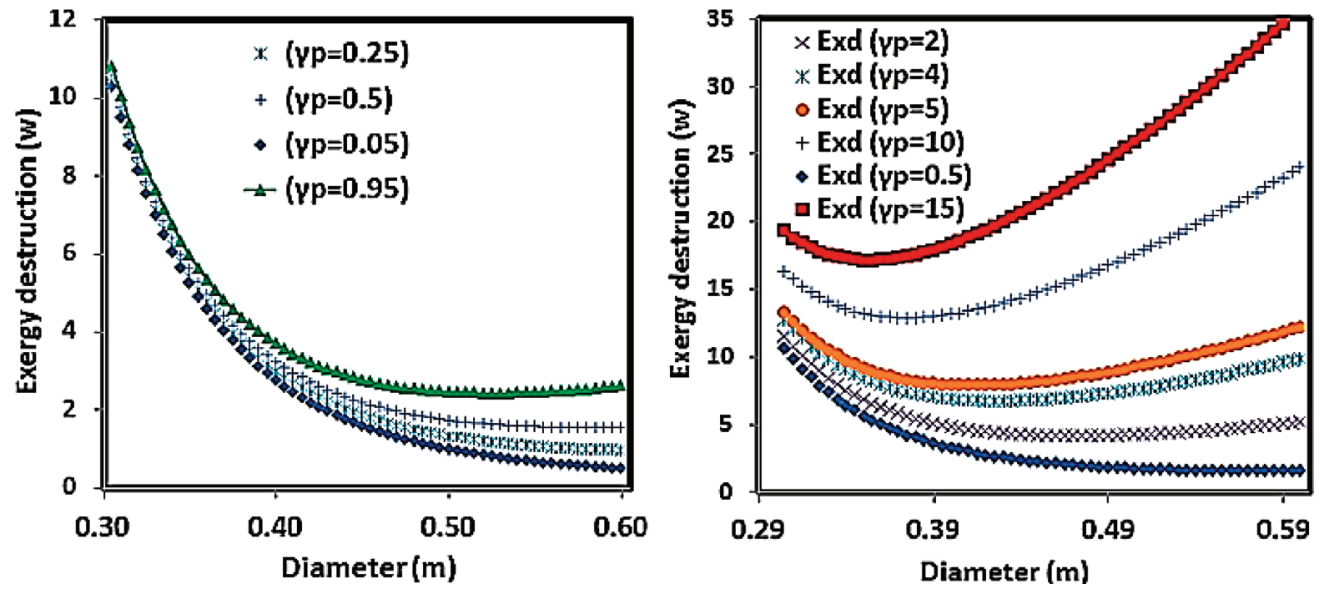

Figure 6: Total exergy destruction for $\gamma_{p}$ ranging from 0.05 to 15 .

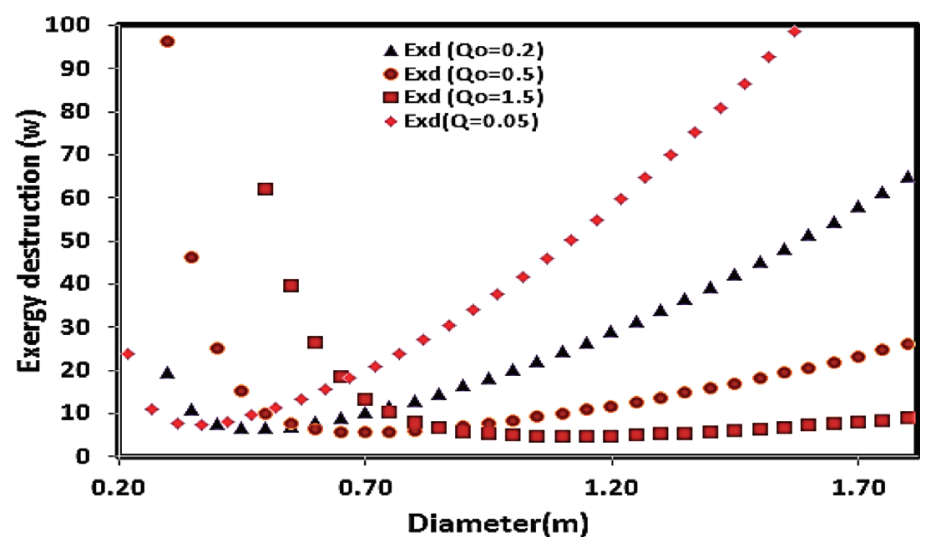

Figure 7: Sensitiveness study of $e x_{d}: \gamma_{p}=4$ and different flow rates.

chemical irreversibilities have been assessed and weighted as a function of the network managers' priority and these irreversibilities have been simultaneously minimized over the pipe diameter. Limits of $\gamma_{p}$ have been determined for different flow rate. For example, for $Q=0.15 \mathrm{~m}^{3} / \mathrm{s}$, it has been shown by the method that the weight coefficient must be confined between 0.6 and 15 in order to have acceptable velocities in the pipes. The originality introduced in this section is the flexibility measures during the procedure of the water distribution network design. These two constraints are shown to be weighable and their weighing limits have been accurately determined for a given flow rate eqn (27).

\section{CONSTRUCTIONS OF THE NETWORKS BY CONSTRUCTAL APPROACH}

First, the method of exergy destruction minimization (based on the new 'reduced exergy destruction' function eqn (23)), coupled with the constructal approach, is used to optimize and to construct two 
water distribution networks. Second, this constructal design has been resumed, by minimizing one of the two irreversible processes while the second is seen as optimization constraint. The construction is performed from a basic elemental network to the overall network obtained after a certain level of construction. Results of the two methods are commented.

4.1 Constructal sequence, method 1: the total exergy destruction minimization method

Consider a k-level of pairing network for both two-branching (N2N) and four-branching (N4N) configurations to cover a given square area. For example $k=2, k=3$ and $k=4$ results in Fig. 8. For each network configuration, the reduced exergy destruction function is written as follows:

$$
e \dot{x}_{d}=\sum_{i=0}^{k}\left(\frac{L_{i} Q_{i}^{n}}{D_{i}^{m}}+\gamma_{p} \frac{L_{i} D_{i}^{2}}{Q_{i}}\right)
$$
By solving the system of equations $\frac{\partial e \dot{x}_{d}}{\partial D_{i}}=0$ we find the optimal diameters scaling laws in the
network eqns (29)-(31).

$$
\begin{gathered}
\left(\frac{D_{k}}{D_{k-1}}\right)=N_{p}^{\frac{n+1}{m+2}} \\
\left(\frac{D_{k}}{D_{0}}\right)=N_{p}^{k \frac{n+1}{m+2}} \\
D_{0}=\left(\frac{m}{2 \gamma_{p}}\right)^{m+2} Q_{0}^{\frac{n+1}{m+2}}
\end{gathered}
$$

where $D_{0}$ is the diameter of terminal branches and $D_{\mathrm{i}}$ is the diameter of the $i^{\text {th }}$ branching level. For this first case, from lower-order constructs to higher ones, the diameters in the upstream of the N4N network increase rapidly than the $\mathrm{N} 2 \mathrm{~N}$ ones according to eqn (30) since the terminal diameters (which do not depend on $N_{p}$ ) have the same expression eqn (31). It means that for higher-order constructs, N4N networks perform very well the exergy destruction minimization than the higherorder N2N networks. Section 5 based on only mechanical irreversibilities minimization under a water quality constraint, performed by Lagrangian multipliers method, confirms these results more explicitly with some offered illustration figures.

4.2 Constructal sequence, method 2: mechanical irreversibilities minimization under a water quality constraint

Another way of optimizing water distribution network is to reduce the exergy destruction to its mechanical contribution which is the most important part (as seen in Section 2.2.3 and in Fig. 5) and consider the chemical contribution as the design constraint. By this way, the problem is reduced to mechanical irreversibility minimization under water quality constraint. Consider the same k-level of pairing network (presented in Section 4.1) for both N2N and N4N configurations (Fig. 8) to cover 
the same given square area. The head losses and residence time in each system (configuration) are given by:

$$
\Delta H=a \cdot \sum_{i=0}^{k} \frac{Q_{i}^{n}}{D_{i}^{m}} \cdot L_{i} \quad ; \quad t=\frac{\pi}{4} \sum_{i=0}^{k} L_{i} D_{i}^{2} / Q_{i}
$$

By the method of Lagrange multipliers, the linear combination of these two relations leads to:

$$
L_{g}=a \cdot \sum_{i=0}^{k} \frac{Q_{i}^{n}}{D_{i}^{m}} \cdot L_{i}+\lambda\left(\frac{\pi}{4} \sum_{i=0}^{k} L_{i} D_{i}^{2} / Q_{i}-t\right)
$$

When we solve equations $\partial L g / \partial D_{\mathrm{i}}=0$ and eliminate $\lambda$, we then obtain:

$$
D_{0}=\sqrt{\frac{4 t Q_{0} / \pi}{L_{0}+\sum_{i=1}^{k} L_{i}\left(\prod_{i=1}^{i} N_{p}\right)^{\left(2 \frac{n+1}{m+2}-1\right)}}} ;\left(\frac{D_{k}}{D_{k-1}}\right)=N_{p}^{\frac{n+1}{m+2}} ; \frac{D_{k}}{D_{0}}=N_{p}^{k \frac{n+1}{m+2}}
$$

These relations represent the optimal diameters distribution through the network. The minimal water head needed of the network upstream is obtained by re-evaluating eqn (32).

$$
H_{1}=H_{0}+a\left(L_{0}+\sum_{i=1}^{k} N_{p}^{\left(n-m \frac{n+1}{m+2}\right) i} L_{i}\right) J_{0}
$$

where $J_{0}$ is $Q_{0}{ }^{n} / D_{0}{ }^{m}, H_{0}$ is the required water head at the downstream of the terminal branch, and $(a, n, m)$ are the Hazen William friction losses parameters for a given pipe material eqn (1).

\section{RESULTS AND DISCUSSION}

\subsection{Comparison based on constructs order}

Through both constructal method 1 and method 2, a law of optimal diameters distribution for each construct has been determined to minimize irreversibilities which are linked to pumping power of the network. For all the constructs, the performances depend strongly on both the number of pairings $N_{p}$ at nodes and the number of pairing levels $k$. Velocities analysis showed for both configurations that, from the first construct to higher-order constructs, the velocities intervals increase as shown in Fig. 9. Especially, velocities intervals $\left|V_{\min }-V_{\max }\right|$ vary faster for N4N lower-order constructs than for $\mathrm{N} 2 \mathrm{~N}$ ones meaning that velocities distribution is more regular for $\mathrm{N} 2 \mathrm{~N}$ than for $\mathrm{N} 4 \mathrm{~N}$. For lowerorder constructs of N4N, the maximal velocities are higher than the maximal velocities of N2N. But they become inferior to N2N's for higher-order constructs. By contrast, minimal velocities have an opposite evolution.

This velocities distribution explains the average velocities evolution shown in Fig. 10. The average velocities of the N2N are inferior to N4N's for lower-order constructs.

These average velocities distribution permit to understand the evolution of the pumping power and the average unit pressure drops through the networks as shown in Fig. 11. 

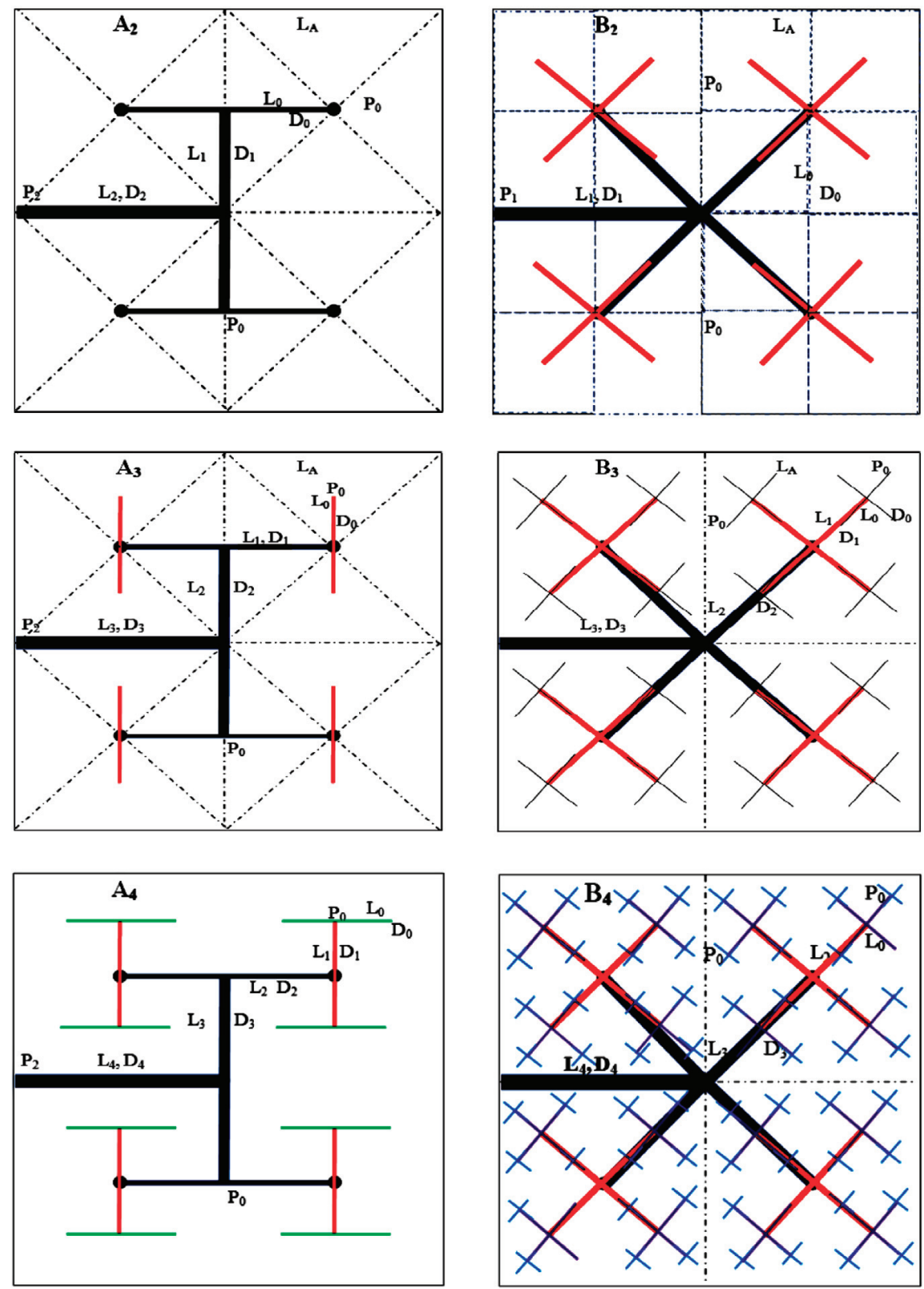

Figure 8: Configuration of constructs level 2, 3 and 4, for $N_{p}=2(\mathrm{~A})$ and $N_{p}=4$ (B).

\subsection{Comparison based on the number of service points (terminal points of the network)}

Another way of comparing these networks is to look at their characteristics in relation with the number of points to be irrigated. From this point of view, the second construct of the N2N networks $(\mathrm{N} 2 \mathrm{~N})_{2}$ can be compared with the first construct of the $\mathrm{N} 4 \mathrm{~N}$ networks $(\mathrm{N} 4 \mathrm{~N})_{1}$ and $(\mathrm{N} 2 \mathrm{~N})_{4}$ to $(\mathrm{N} 4 \mathrm{~N})_{2}$ 


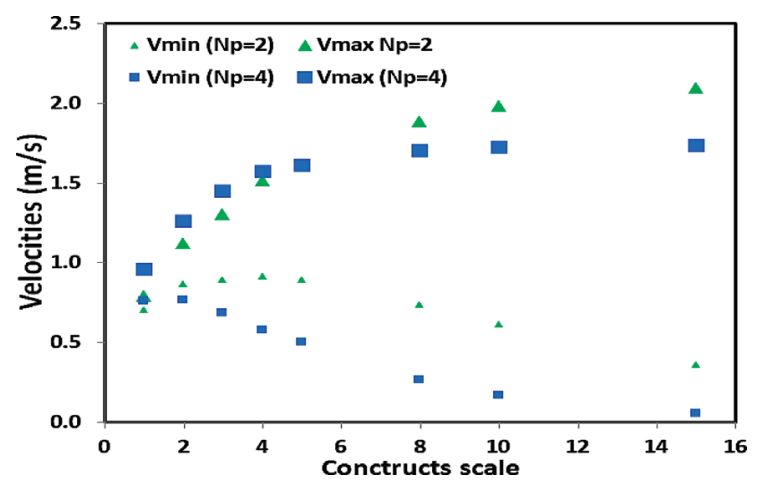

Figure 9: Maximum and minimum velocities in N2N and N4N networks.

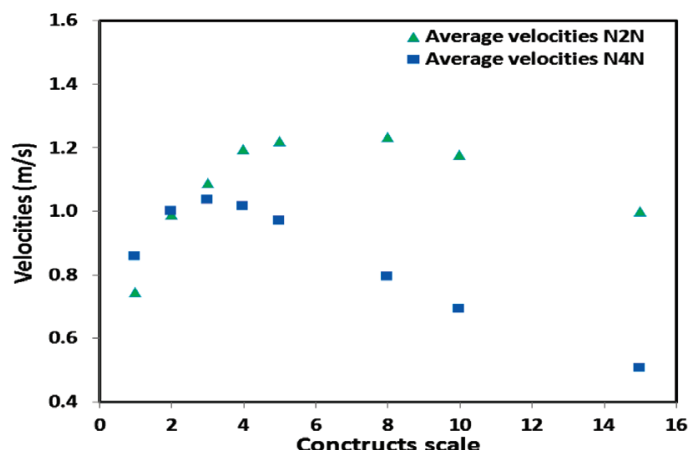

Figure 10: Average velocities through N2N and N4N networks.
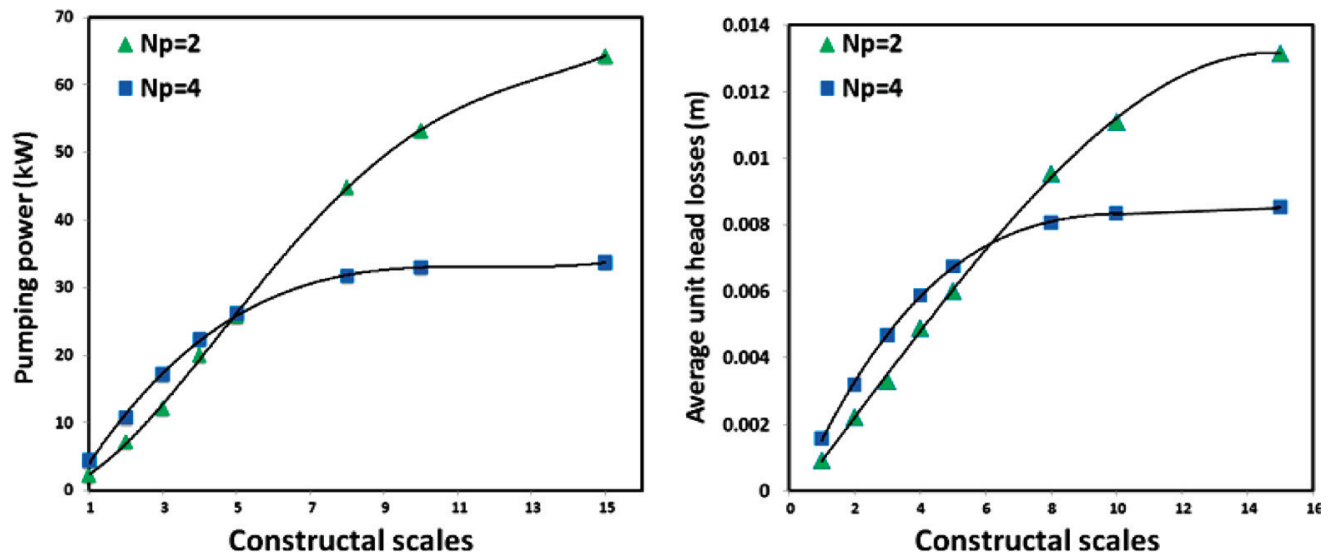

Figure 11: Pumping power and average unit head losses over constructs scales. 
and so forth. Figure 12 shows that for a same number of points to be irrigated, N2N networks need a pumping power close to twice the pumping power required for N4N networks.

Figure 13 confirms this fact by showing that the average unit head losses in N2N networks represent the double of N4N's ones. Say another way, from the operating cost point of view, N4N networks are better than $\mathrm{N} 2 \mathrm{~N}$ networks.

The two methods ( 1 and 2 ) presented here are fundamentally the same since they are both based on two objective functions which parameters make them equivalent. Indeed, in the method 1 , the $\gamma_{p}$ weight plays the same role as the constraint of residence time $t$ described in method 2. They represent the water quality control tools. Nevertheless, even if these two methods have proven to be equivalent, method 1 seems to be very adapted for cases where flexibility is required.

\section{DOMAINS OF APPLICATION}

Figure 14 shows that the average unit head losses are inferior for N4N to N2N when higher-order constructs are considered.

Besides, average velocities in $\mathrm{N} 4 \mathrm{~N}$ are inferior to N2Ns' ones and the number of irrigated points is the highest; meaning that $\mathrm{N} 4 \mathrm{~N}$ can irrigate well a surface where points are very concentrated.

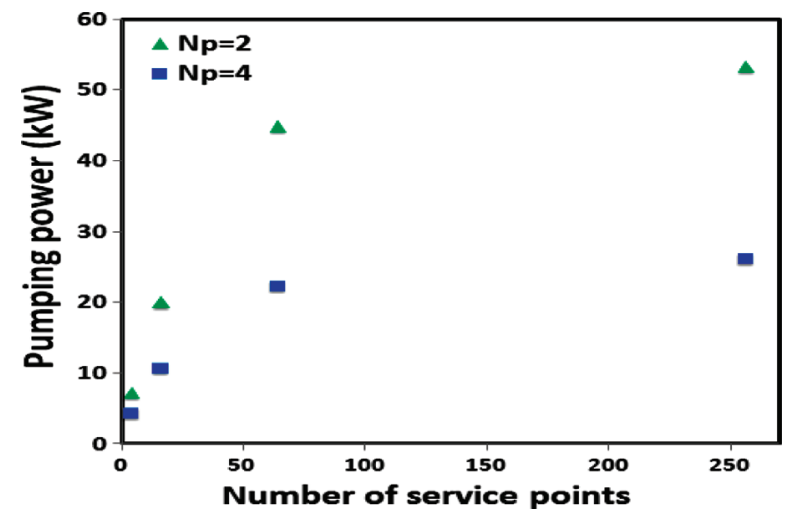

Figure 12: Pumping evolution related to the number of irrigated points.

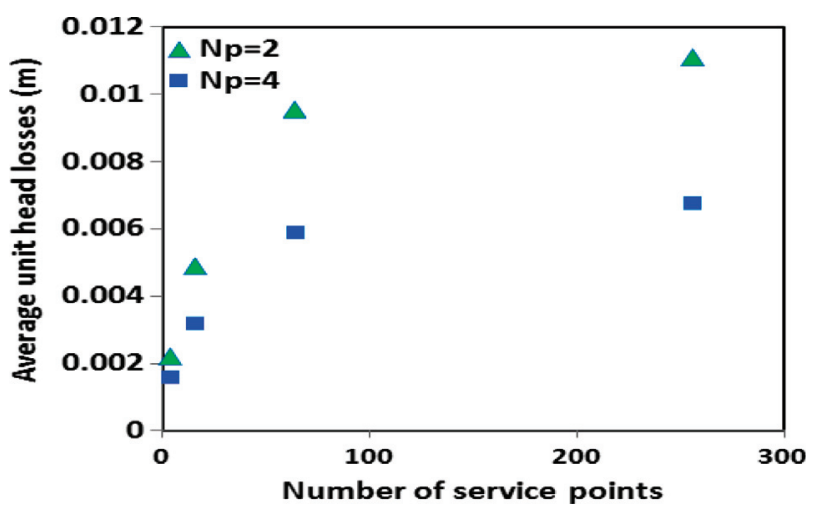

Figure 13: Average unit head losses related to the number of irrigated points. 


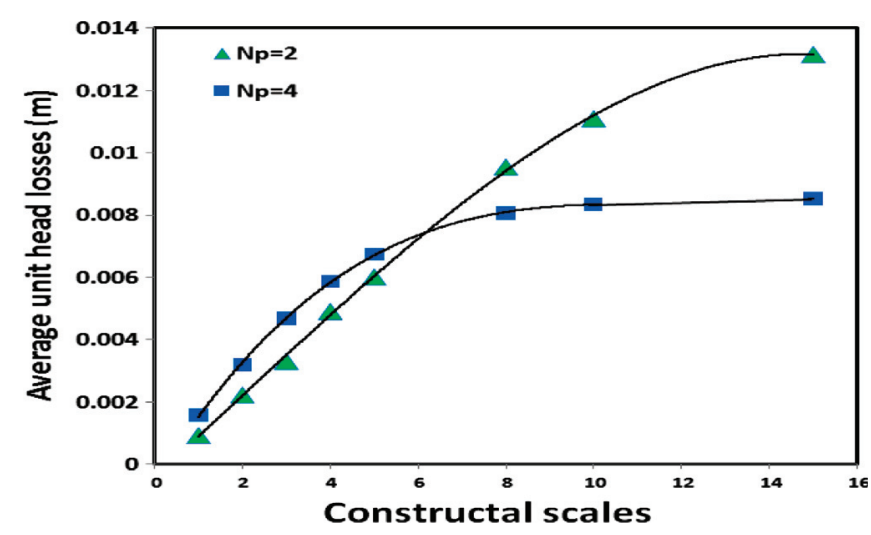

Figure 14: Average unit head losses for different constructal scales.

These criteria of low velocities and a large number of points to be irrigated are precisely met in the domains of irrigation in agriculture, according to literature on irrigation technique [19-21]. For these reasons, the N4N networks considered in higher-order constructs seems to be suitable for irrigation. But for drinking water systems, the water quality evolution is well known according to Delahaye et al. [22] and Clarck et al. [23] to be degradable with the residence time. That is why it is revelant from an environmental protection point of view (good control of water quality) to consider the N2N networks as the most adapted to drinking water distribution systems for their good flow velocities distribution through the networks.

\section{CONCLUSION}

This study showed that in the construction of tree-shaped network for water distribution, the method of exergy destruction minimization is equivalent to minimizing mechanical irreversibility (that are equal to pumping power) under a water quality constraint. For both phenomena appearing in the network (energy consumption and the fluid quality degradation), this paper offers a new interesting route of optimizing the system either by the exergy destruction minimization or one phenomenon minimization under a constraint that takes into account the second phenomenon.

Economic aspects, water demands fluctuation and topography variation were not considered and minor losses were neglected here but for further investigations they could be included to be close to a realistic water distribution network.

This study also showed that for the improvement of performances in a distribution network it is important to focus on the design the network rather than enhancing only its transport properties. All the optimized geometries have the same total mass (since the water residence time $t$ which formula is, according to Tondeur et al. [24], proportional to total volume of ducts is fixed) meaning they are roughly identical from the investment cost point of view. Consequently, the best one should be the one which suits the use made of it as discussed above in discussion section and which performs pumping power minimization.

Once again, the constructal approach has proven to be a powerful design method that can be used, based on various methodologies, to perform flow systems design under any given constraint. The approach presented in this paper should be seen as an introduction to reactive flow architectures design by constructal approach and a contribution to the design problems under environmental constraints. 


\section{NOMENCLATURE}

C chemical concentration, $\mathrm{kg} / \mathrm{l}$

$D$ diameter, $\mathrm{m}$

$e \dot{x}_{d}$ exergy destruction, $\mathrm{W}$

ex exergy of the system, $\mathrm{W}$

$g$ gravitational acceleration, $\mathrm{m} / \mathrm{s}^{2}$

$H$ water head, m

$h_{i} \quad$ total molar enthalpy, $\mathrm{J} / \mathrm{mole}$

$J$ unit head losses, mCE

$k$ chlorine residual decay, /s

$L$ pipe length, $\mathrm{m}$

$m$ mass, $\mathrm{kg}$

$M_{\mathrm{e}} \quad$ water molar mass, $\mathrm{kg} / \mathrm{mole}$

$Q \quad$ volumetric flow rate, $\mathrm{m}^{3} / \mathrm{s}$

$\dot{q}_{0} \quad$ heat losses exchanged with surrounding, $\mathrm{W}$

$R$ ideal gas constant, $\mathrm{J} / \mathrm{kg} / \mathrm{K}$

$S$ entropy, J/K

$t \quad$ water residence time, $\mathrm{s}$

$T_{0}$ ambient temperature, $\mathrm{K}$

$U$ internal energy, $\mathrm{J}$

$v_{s} \quad$ specific volume, $\mathrm{m}^{3} / \mathrm{kg}$

Greek symbols

$\Delta C$ concentration variation, $\mathrm{kg} / \mathrm{l}$

$\Delta H$ head losses, $\mathrm{m}$

$\rho \quad$ water density, $\mathrm{kg} / \mathrm{m}^{3}$

$\lambda$ friction factor

Subscripts

Ch chemical

$C_{H W}$ Hazen William head losses coefficient for a given material

Mec mechanical

Max maximal

Min minimal

Re Reynolds number

$y_{i} \quad$ molar fraction of the constituent $i$

1 input

2 output

\section{REFERENCES}

[1] Bejan, A., Constructal-theory network of conducting paths for cooling a heat generating volume. International Journal of Heat Mass Transfer, 40(4), pp. 799-816, 1997. doi: http://dx.doi. org/10.1016/0017-9310(96)00175-5

[2] Azoumah, Y., Mazet, N. \& Neveu, P., Constructal network for heat and mass transfer in a solidgas reactive porous medium. International Journal of Heat Mass Transfer, 47(14-16), pp. 2961-2970, 2004. doi: http://dx.doi.org/10.1016/j.ijheatmasstransfer.2004.03.022 
[3] Bejan, A. \& Lorente, S., Constructal tree-shaped flow structures. Applied Thermal Engineering, 27(4), pp. 755-761, 2007. doi: http://dx.doi.org/10.1016/j.applthermaleng.2006.10.008

[4] Bejan, A. \& Lorente, S., Design with Constructal Theory, Wiley: Hoboken, 2008. doi: http:// dx.doi.org/10.1002/9780470432709

[5] Kerneïs, A., Nakache, F., Deguin, A. \& Feinberg, M., The effects of water residence time on the biological quality in a distribution network. Water Research, 29(7), pp. 1719-1727, 1995. doi: http://dx.doi.org/10.1016/0043-1354(94)00323-Y

[6] Bieupoude, P., Azoumah, Y. \& Neveu, P., Environmental optimization of tree-shaped water distribution networks. Presented at the 5th International Conference on Comparing Design in Nature with Science and Engineering, accepted for publication in the Proceedings of the 6th International Conference on Sustainable Water Resources Management, WIT Press: Riverside, California, 2011.

[7] Prigogine, I., Introduction to Thermodynamics of Irreversible Process, Wiley: New York, 1962.

[8] Carlier, M., Hydraulique Générale et appliquée, Eyrolles: Paris, 1972.

[9] Lahiouel, Y., Haddad, A. \& Chaoui, K., Evaluation of head losses in fluid transportation networks. Sciences \& Technologie, 23, pp. 89-94, 2005.

[10] Walski, T., Chase, D. \& Savic, D., Water Distribution Modeling, Heasted Method, Heasted Press: USA, 2001.

[11] Rossman, L., Clark, R. \& Grayman, W.M., Modeling chlorine residual in drinking-water distribution system. Journal of Environmental Engineering, 120(4), pp. 803-820, 1994. doi: http://dx.doi.org/10.1061/(ASCE)0733-9372(1994)120:4(803)

[12] World Health Organization, Guidelines for Drinking-Water Quality, vol. 2, 2nd edn, Health Criteria and Other Supporting Information. WHO, Mastercom/Wiener Verlag, 1996.

[13] Crittenden, J., Trussell, R., Hand, D., Howe, K. \& Tchobanoglous, G., Water Treatment, Principles and Design, 2nd edn, John Wiley \& Sons: New York, 2005.

[14] Powell, J., Hallam, N., West, J., Forster, C. \& Simms, J., Factors which control bulk chlorine decay rates. Water Research, 34(1), pp. 117-126, 2000. doi: http://dx.doi.org/10.1016/S00431354(99)00097-4

[15] Rodriguez, M., Milot, J., Sérodes, J. \& Pacaud, A., Estimation of bench-scale chlorine decay in drinking water using nth order kinetic and back propagation neural network models. Water Quality Research, 37(3), pp. 613-635, 2001.

[16] Environmental Protection Agency (EPA), National primary drinking water regulations: disinfectants and disinfection by products rule; Final rule. Federal Register, 63(241), pp. 69390-69476, 1998.

[17] Torrijos, M. \& Moletta, R., Winery wastewater depollution by sequencing batch reactor. Water Science and Technology, 35(1), pp. 249-257, 1997. doi: http://dx.doi.org/10.1016/S02731223(96)00903-1

[18] Montangero, A. et al., Optimising water and phosphorus management in the urban environmental sanitation system of Hanoi. Vietnam Science of the Total Environment, 384(1-3), pp. 55-66, 2007. doi: http://dx.doi.org/10.1016/j.scitotenv.2007.05.032

[19] Zella, L. \& Kettab, A., Optimisation d'un réseau de micro-irrigation = Optimising a microirrigation network. Sécheresse, 14(3), pp. 189-194, 2003.

[20] Calejoa, M., Lamaddalenab, N., Teixeirac, J. \& Pereirac, L., Performance analysis of pressurized irrigation systems operating on-demand using flow-driven simulation models. Agricultural Water Management, 95(2), pp. 154-162, 2008. doi: http://dx.doi.org/10.1016/j. agwat.2007.09.011

[21] De La Bouëre, J., Micro-irrigation, bases et principes DVD réf MA2602, Lavoisier 2000-2010, 2006. 
92 P. Bieupoude et al., Int. J. of Design \& Nature and Ecodynamics. Vol. 7, No. 1 (2012)

[22] Delahaye, E., Welté, B., Levi, Y., Leblon, G. \& Montiel, A., An ATP-based method for monitoring the microbiological drinking water quality in a distribution network. Water Research, 37(15), pp. 3689-3896, 2003. doi: http://dx.doi.org/10.1016/S0043-1354(03)00288-4

[23] Clarck, R., Rossman, L. \& Wymer, L., Modeling distribution system water quality: regulatory implications. Journal of Water Resources Planning and Management, 121(66), pp. 423-428, 1995. doi: http://dx.doi.org/10.1061/(ASCE)0733-9496(1995)121:6(423)

[24] Tondeur, D., Fan, Y. \& Luo, L., Constructal optimization of arborescent structures with flow singularities. Chemical Engineering Science, 64(18), pp. 3968-3982, 2009. doi: http://dx.doi. org/10.1016/j.ces.2009.05.052 